\title{
ANALYSIS OF FIBREOPTIC BRONCHOSCOPY STUDIES AT A TERTIARY HEALTH CARE CENTER IN MALWA REGION
}

Satish Motiwale ${ }^{1}$, Ravindra Chordiya ${ }^{2}$, Prakash Joshi ${ }^{3}$

\section{HOW TO CITE THIS ARTICLE:}

Satish Motiwale, Ravindra Chordiya, Prakash Joshi. "Analysis of Fibreoptic Bronchoscopy Studies at a Tertiary Health Care Center in Malwa Region." Journal of Evolution of Medical and Dental Sciences 2014; Vol. 3, Issue 09, March 3; Page: 2179-2181, DOI: $10.14260 /$ jemds/2014/2123

ABSTRACT: Bronchoscopy is an invasive test done with intention of diagnosing and treating complex pulmonary diseases. As a tertiary referral center in central India we received several such cases and analyzed 200 Bronchoscopy performed over 1 year. The indications of bronchoscopy are numerous and usually based on the presence of respiratory symptoms and abnormal chest radiograph or both. Common indications include peripheral pulmonary masses, hemoptysis, chronic cough, pleural effusion, recent or unresolved pneumonia, pulmonary tuberculosis, and lung collapse. In areas with high prevalence of pulmonary tuberculosis, bronchoscopy and bronchoscopic lung biopsy were found to be useful for diagnosis of pulmonary tuberculosis especially in the absence of radiological infiltration and negative sputum smears for acid fast bacilli. Several studies have shown that bronchoscopy is a safe procedure that carries very low mortality rate that ranges from $0 \%$ to $0.1 \%$.

KEYWORDS: Bronchoscopy.

\section{AIMS AND OBJECTIVES:}

- To analyze the Indication, retrospectively with an intent to streamline and optimize our future performance and decrease patients morbidity \& mortality.

- To assess the safety of Fibre Optic Bronchoscopy Procedure

- To correlate the Bronchoscopic findings with radiology and smoking status of the subjects.

\section{MATERIALS AND METHODS:}

- Fiber optic Bronchoscope of Olympus make both adult and pediatric were used in local and general Anesthesia in Dept. of Respiratory Medicine SAIMS medical college \& PGI, Indore in admitted \& referred patients. Pre procedure Informed consent and basic hematology workup done before procedure. The Data Was Collected From August 2011 To July 2012.

RESULTS: A total of 200 cases underwent bronchoscopy over the mentioned period,

The most common indication of Bronchoscopy clinically were,

1. Chronic cough

2. Hemoptysis

3. Hoarseness of voice

The most common indication of Bronchoscopy radiologically were,

1. Collapse

2. Mass Lesions

3. Lobar Consolidation 
The most common bronchoscopy findings were,

1. Malignancy -44

2. Tubercular -40

3. Normal Study -66

4. Inflammatory lesion -50

The most commonly observed complications

\section{During procedure}

Hypoxia - 45

Irritability -130

Bleeding - 58

Cough - 45

\section{Post procedure}

Hypoxia - 89

Irritability - 18

Bleeding -5

Cough - 78

DISCUSSION: The safety of FFB has been documented from different parts of the world.1,2

The mortality rate due to the procedure is exceedingly low (from 0-0.01\%).

Although Dreisen et al reported a higher mortality rate among their 205 FFBs of $0.5 \%$. The mortality obviously depends on the scrutiny of selection of patients for the procedure and the experience of the bronchoscopist and the facilities available.

Our rate of major complications compares favorably with those from other studies.1, 2,3 Our pneumothorax rate (4\%) compares with their pneumothorax rates of $0.16 \%, 2 \%$ and $30 \%$ (with hemorrhage). We had no pulmonary hemorrhage or respiratory failure compared with a rate of $0.32 \%$ of these two major complications 16 and a $5 \%$ rate of major complications by Dreisen et al. ${ }^{4}$

Minor complications in our study included hypoxemia and sinus tachycardia as the most common followed by bronchospasm. Our rate of hypoxemia (14\%) and tachycardia (5\%) compared with (42\%) and (15.5\%) by Davies et al. ${ }^{1,2}$

Furthermore, hypoxia has been a recognized complication both of transoral and transnasal FFB for at least 25 years. ${ }^{2}$ Dysrhythmias have also frequently been described, ${ }^{2,3}$ we had none.

Bronchospasm may be prevented by the inhalation of four puffs of $0.02-\mathrm{mg}$ ipratropium bromide 15 minutes before FFB. ${ }^{4}$ Our mortality rate was $0 \%$; but a more realistic figure of $0.01 \%$ was quoted by Credle et al1 after reviewing 24, 521 FFBs performed by 250 physicians. This although absolute contraindications to bronchoscopy are limited to life-threatening arrhythmia or refractory hypoxemia with relative contraindications of increase risk of bleeding during biopsy. ${ }^{2}$

Our top four indications for FFB were pulmonary infiltration in the chest x-ray, lung mass, hemoptysis and interstitial fibrosis in that order, which constituted $75 \%$ of the total. This compares with $52 \%$ Fibs performed for suspected infection in another study. ${ }^{1}$ Of the 124 patients in our series, about one third (38 patients=31\%) had FFB for suspected TB. The diagnosis was confirmed in 28 patients either by TB granuloma in the histology of transbronchial or endobronchial biopsy (12 
patients) and/or positive AFB smear or culture in the lavage fluid (16 patients). This compares with $32.5 \%$ bronchoscopic diagnostic yield for tuberculosis in another study. ${ }^{2}$ The diagnostic role of FFB in pulmonary tuberculosis has been established especially in countries where the prevalence of tuberculosis is moderately high such as Thailand and Saudi Arabia. ${ }^{2}$

The yield of FFB in our series was fairly high and the correlation with radiologically findings and histology was good. This was shown by the fact that of the 38 patients suspected of having TB or other infections, on radiological or clinical grounds, 28 had proven TB by bronchoscopic techniques i.e. biopsy or acid fast bacilli (AFB) in the lavage fluid.

Furthermore, of the 46 patients who had a lung mass radiologically or hemoptysis, 31 had bronchial carcinoma following FFB. None of our patients underwent an open lung biopsy, as the great majority of them were diagnosed by mean short of open lung biopsy. The well-established link between smoking and lung cancer has been highlighted again, as $84 \%$ of lung cancer patients in this series were either smokers or ex-smokers.

In conclusion, this study shows that FFB is a safe and a useful procedure in diagnosing many chest diseases when performed under appropriate conditions and that it has a high yield and a low rate of complications.

\section{REFERENCES:}

1. Credle WF, Smidly JF, Elliot RC. Complications of fiberoptic bronchoscopy. Am rev Resp Dis 1974; 109: 67-72.

2. Pue CA, Pacht ER. Complications of fiberoptic bronchoscopy at a university hospital. Chest 1995; 107:430-432.

3. Dreisen RB, Albert RK, Talley PA, Kryger MH, Seoggin $\mathrm{CH}$, Zwillich CW. Flexible fiberoptic bronchoscopy in teaching hospital. Chest 1978; 74: 144-149.

4. Pereira W, Kovant DM, Snider GL. A prospective cooperative study of complications following flexible fiberoptic bronchoscopy. Chest 1978; 73: 813-816.

\section{AUTHORS:}

1. Satish Motiwale

2. Ravindra Chordiya

3. Prakash Joshi

\section{PARTICULARS OF CONTRIBUTORS:}

1. Associate Professor, Department of Chest Medicine, Sri Aurobindo Institute of Medical Sciences \& Post Graduate Institute, Indore.

2. Professor and HOD, Department of Chest Medicine, Sri Aurobindo Institute of Medical Sciences \& Post Graduate Institute, Indore.

3. Senior Registrar, Department of Chest Medicine, Sri Aurobindo Institute of Medical Sciences \& Post Graduate Institute, Indore.

\section{NAME ADDRESS EMAIL ID OF THE} CORRESPONDING AUTHOR:

Dr. R. Chordiya,

Department of Respiratory Diseases, SAMC \& PGI,

Indore.

E-mail: deptoftbcsaims@yahoo.in

Date of Submission: 09/02/2014. Date of Peer Review: 10/02/2014. Date of Acceptance: 19/02/2014. Date of Publishing: 26/02/2014. 